\title{
Frequency of $R N F 213$ p.R4810K, a susceptibility variant for moyamoya disease, and health characteristics of carriers in the Japanese population
}

\author{
Yang Cao $^{1} \cdot$ Hatasu Kobayashi ${ }^{1} \cdot$ Takaaki Morimoto $^{1,2} \cdot$ Risako Kabata $^{1}$ • \\ Kouji H. Harada ${ }^{1} \cdot$ Akio Koizumi $^{1}$
}

Received: 2 June 2016/Accepted: 23 June 2016/Published online: 30 June 2016

(C) The Japanese Society for Hygiene 2016

\begin{abstract}
Objectives RNF213 p.R4810K is a founder polymorphism that confers genetic susceptibility to moyamoya disease in East Asia. Only a few studies have investigated the symptoms and disease histories of RNF213 p.R4810K carriers in Japan. This study investigated the frequency of $R N F 213$ p.R4810K in the general Japanese population and the health characteristics of the carriers.

Methods Through a health-promotion campaign in the city of Uji, Japan, 519 subjects (120 males and 399 females) of the general Japanese population were genotyped for $R N F 213$ p.R $4810 \mathrm{~K}$ and interviewed to determine health characteristics.

Results Nine RNF213 p.R4810K heterozygous carriers (GA genotype) and no RNF213 p.R4810K homozygous carriers (AA genotype) were found among the 519 individuals. The estimates of the genotypes and allele frequencies for $R N F 213$ p.R4810K were 1.73 and $0.87 \%$, respectively. There were no obvious differences in age, gender ratio, body mass index, hypertension, dyslipidemia, diabetes, kidney disease, liver disease, heart
\end{abstract}

Akio Koizumi

koizumi.akio.5v@kyoto-u.ac.jp

1 Department of Health and Environmental Sciences, Kyoto University Graduate School of Medicine, Yoshida, Sakyo-ku, Kyoto 606-8501, Japan

2 Department of Neurosurgery, Kyoto University Graduate School of Medicine, Shogoin Kawahara, Sakyo-ku, Kyoto 606-8507, Japan disease, or drinking or smoking habits between carriers and non-carriers. Interestingly, one patient with moyamoya disease was found among the nine RNF213 p.R4810K carriers.

Conclusions This study showed the genotypes and allele frequencies of $R N F 213$ p.R4810K in the general Japanese population to be similar to results of previous reports.

Keywords $R N F 213$ - p.R4810K · Moyamoya disease . General population · Japan

\section{Introduction}

Moyamoya disease (MMD) is an idiopathic vascular disorder of intracranial arteries [1]. MMD occurs with the highest prevalence in East Asian countries, including Japan, Korea, and China, compared with other countries worldwide [2-5]. Recently, RNF213 has been identified as a susceptibility gene for MMD. The p.R4810K polymorphism (c.14429G > A: rs112735431) in RNF213 has a strong association with the disease in Japanese, Korean, and Chinese MMD patients [6, 7], and the frequency of p.R4810K in the general Japanese population has been estimated at 1.4-2.7\% [6-11]. However, no studies have investigated symptoms and disease histories of RNF213 p.R4810K carriers in the general Japanese population, except for one report that tested the association of RNF213 p.R4810K with blood pressure [10]. In this study, our major objective was to investigate the frequency of $R N F 213$ p.R4810K in 519 Japanese individuals from the city of Uji, Japan, and to determine the health characteristics of the carriers. We aimed to determine whether RNF213 p.R4810K carriers have specific health problems. 


\section{Materials and methods}

\section{Study population}

A total of 519 Japanese individuals were recruited between 2008 and 2015 from the participants of a health-promotion campaign in Uji, Kyoto Prefecture, Japan. For each participant, we chose the latest data collected in the healthpromotion campaign for analysis. Participant information, including age, sex, history of hypertension (HT), dyslipidemia (DL), diabetes, heart disease, liver disease, kidney disease, any other disease, and drinking and smoking habits, were collected by interview. These diseases were defined as self-reported physician diagnosis or pharmaceutical treatment. Body weight was measured in light clothes on a scale, and height was measured with a stadiometer. Body mass index [BMI, weight $(\mathrm{kg}) /$ height $\left(\mathrm{m}^{2}\right)$ ] was calculated. Ethical approval for this study was given by the Institutional Review Board and Ethics Committee of Kyoto University School of Medicine, Kyoto, Japan (G182 and G342). Written informed consent was obtained from all individual participants included in this study.

\section{DNA extraction and genotyping of RNF213 p.R4810K}

Genomic DNA was extracted from blood samples using a QIAamp DNA Blood Mini Kit (QIAGEN, Germany) according to the manufacturer's protocol. The DNA was stored at $-20{ }^{\circ} \mathrm{C}$ until analysis. Genotyping of p.R4810K was conducted using a TaqMan probe (Custom TaqMan ${ }^{\circledR}$ SNP Genotyping Assays; Applied Biosystems, Foster City, CA, USA) and a 7300/7500 Real-Time PCR System (Applied Biosystems) as described previously [7].

\section{Statistical analyses}

All data analysis was carried out using JMP pro version 11.2.0 (SAS, Cary, NC, USA). Continuous variables (age, sex, and BMI) are presented as the mean \pm standard deviation (SD) and age was compared using Student's $t$ test. Categorical variables (HT, DL, diabetes, drinking, smoking, kidney disease, liver disease, and heart disease) are presented as proportions. $P$ values $<0.05$ were considered statistically significant.

\section{Results}

The demographic characteristics and frequency of $R N F 213$ p.R4810K among the participants of this study are shown in Table 1. There were 120 men and 399 women. Among
Table 1 Demographic characteristics and frequency of RNF213 p.R4810K in study participants

\begin{tabular}{ll}
\hline Number, $n$ & 519 \\
Age (years), mean \pm SD & $62.99 \pm 15.47$ \\
Male sex, $n(\%)$ & $120(23.12)$ \\
Hypertension, $n(\%)$ & $119(22.93)$ \\
Dyslipidemia, $n(\%)$ & $46(8.86)$ \\
Diabetes, $n(\%)$ & $29(5.59)$ \\
Drinking, $n(\%)$ & $272(52.40)$ \\
Smoking, $n(\%)$ & $97(18.69)$ \\
Kidney disease, $n(\%)$ & $23(4.43)$ \\
Liver disease, $n(\%)$ & $29(5.59)$ \\
Heart disease, $n(\%)$ & $58(11.18)$ \\
BMI (kg/m $\left.{ }^{2}\right)$, mean \pm SD & $21.96 \pm 3.30$ \\
Genotype & \\
GG & 510 \\
GA & 9 \\
AA & 0 \\
Carrier frequency of p.R4810K (\%) & 1.73 \\
Allele & \\
G & 1029 \\
A & 9 \\
Minor allele frequency $(\%)$ & 0.87 \\
\hline
\end{tabular}

$B M I$ body mass index, $S D$ standard deviation

the 519 individuals, nine $(1.73 \%)$ had the GA genotype for p.R4810K and none had the AA genotype. We compared the health characteristics of individuals with and without p.R4810K (Table 2). There were no significant differences in age between GG and GA groups. Furthermore, no obvious differences were observed in gender ratio, BMI, HT, DL, diabetes, kidney disease, liver disease, heart disease, or drinking or smoking habits between GG and GA groups. We confirmed that there was no blood-relationship among the nine individuals with p.R4810K. Interestingly, one patient with MMD was found among the nine RNF213 p.R4810K carriers, while none were found among the noncarriers. The MMD patient was a 43-year-old female who has no history of HT, DL, diabetes, kidney disease, liver disease, and heart disease, and drinking and smoking habits.

\section{Discussion}

This study showed that the genotypes and allele frequencies of RNF213 p.R4810K in the general Japanese population were 1.73 and $0.87 \%$, respectively, which are similar to frequencies previously reported [6-11]. Geographic distribution of RNF213 p.R4810K in East Asian 
Table 2 Characteristics of the study population according to RNF213 p.R4810K variant genotypes

\begin{tabular}{|c|c|c|c|}
\hline & GG & GA & $P$ value \\
\hline Number, $n$ & 510 & 9 & \\
\hline $\begin{array}{l}\text { Age (years), } \\
\text { mean } \pm \mathrm{SD} \text { (range) }\end{array}$ & $63.0 \pm 15.4(21-91)$ & $\begin{array}{l}62.0 \pm 19.2 \\
(34-88)\end{array}$ & 0.9 \\
\hline Male sex, $n(\%)$ & $119(23.3)$ & $1(11.1)$ & \\
\hline $\begin{array}{l}\mathrm{BMI}\left(\mathrm{kg} / \mathrm{m}^{2}\right) \\
\text { mean } \pm \mathrm{SD}\end{array}$ & $22.0 \pm 3.3$ & $21.2 \pm 3.2$ & \\
\hline Hypertension, $n(\%)$ & $118(23.1)$ & $1(11.1)$ & \\
\hline Dyslipidemia, $n(\%)$ & $46(9.0)$ & $0(0)$ & \\
\hline Diabetes, $n(\%)$ & $29(5.7)$ & $0(0)$ & \\
\hline Kidney disease, $n(\%)$ & $22(4.3)$ & $1(11.1)$ & \\
\hline Liver disease, $n(\%)$ & $29(5.7)$ & $0(0)$ & \\
\hline Heart disease, $n(\%)$ & $58(11.4)$ & $0(0)$ & \\
\hline Drinking, $n(\%)$ & $269(52.8)$ & $3(33.3)$ & \\
\hline Smoking, $n(\%)$ & $96(18.8)$ & $1(11.1)$ & \\
\hline Other diseases, $n^{\mathrm{a}}$ & $\begin{array}{l}\text { Cancer, } 15 \text {; tumor, } 6 \text {; cerebral infarction, } 6 \text {; anemia, } 3 \text {; uterine fibroids, } 2 \text {; stomach ulcer, } 6 \text {; } \\
\text { vestibular neuritis, } 1 \text {; duodenal ulcers, } 3 \text {; hemorrhoids, } 1 \text {; tuberculosis, } 3 \text {; connective } \\
\text { tissue disease, } 2 \text {; allergies, } 64 \text {; joint disease, } 5 \text {; hypothyroidism, } 5 \text {; hyperuricemia, } 1 \text {; } \\
\text { reflux esophagitis, } 1 ; \text { osteoporosis, } 5 \text {; cataract, } 4 \text {; non-tuberculous bacteria disease, } 1 \text {; } \\
\text { macroglobulinemia, } 1\end{array}$ & $\begin{array}{l}\text { MMD, 1; } \\
\text { osteoporosis, } \\
1 ; \text { connective } \\
\text { tissue } \\
\text { diseases } 1\end{array}$ & \\
\hline
\end{tabular}

Continuous variables are expressed as mean \pm SD. Categorical variables are expressed as percentages

$B M I$ body mass index, $S D$ standard deviation, $M M D$ moyamoya disease

${ }^{\text {a }}$ The number of participants who has/had the disease is shown

Table 3 Geographic distribution of RNF213 p. R4810K in East Asian populations

\begin{tabular}{|c|c|c|c|c|c|c|c|c|}
\hline \multirow[t]{2}{*}{ Country } & \multirow[t]{2}{*}{ Location } & \multicolumn{3}{|c|}{ Genotype of p.R4810K } & \multirow[t]{2}{*}{ Sample size } & \multirow[t]{2}{*}{ Carrier frequency $\%$} & \multirow[t]{2}{*}{ MAF \% } & \multirow[t]{2}{*}{ References } \\
\hline & & GG & GA & AA & & & & \\
\hline \multirow[t]{10}{*}{ Japan } & Uji city & 510 & 9 & 0 & 519 & 1.73 & 0.87 & This study \\
\hline & Mainland & 1339 & 32 & 3 & 1374 & 2.55 & 1.38 & [9] \\
\hline & Mainland & 374 & 9 & 1 & 384 & 2.60 & 1.43 & [7] \\
\hline & Okinawa & 98 & 2 & 0 & 100 & 2.00 & 1.00 & [9] \\
\hline & Nyukawa & 959 & 23 & 2 & 984 & 2.54 & 1.39 & {$[10]$} \\
\hline & Noshiro & 2432 & 11 & 0 & 2443 & 0.45 & 0.23 & [10] \\
\hline & Field $^{\mathrm{a}}$ & 857 & 23 & 1 & 881 & 2.72 & 1.44 & [10] \\
\hline & Unknown & 423 & 6 & 0 & 429 & 1.40 & 0.70 & [6] \\
\hline & Unknown & 278 & 5 & 0 & 283 & 1.77 & 0.89 & [8] \\
\hline & Unknown & 108 & 2 & 0 & 110 & 1.81 & 0.92 & [11] \\
\hline \multirow[t]{4}{*}{ China } & Northern part & 339 & 3 & 0 & 342 & 0.88 & 0.44 & [9] \\
\hline & Southern part & 243 & 2 & 0 & 245 & 0.82 & 0.41 & [9] \\
\hline & Unknown & 148 & 2 & 0 & 150 & 1.33 & 0.67 & [7] \\
\hline & Unknown & 505 & 2 & 0 & 507 & 0.39 & 0.20 & [13] \\
\hline \multirow[t]{4}{*}{ Korea } & Mainland & 223 & 6 & 0 & 229 & 2.62 & 1.33 & [9] \\
\hline & Jeju-do & 63 & 2 & 0 & 65 & 3.08 & 1.56 & [9] \\
\hline & Unknown & 217 & 6 & 0 & 223 & 2.69 & 1.35 & [7] \\
\hline & Unknown & 1479 & 37 & 0 & 1516 & 2.44 & 1.24 & [12] \\
\hline
\end{tabular}

MAF minor allele frequency

${ }^{a}$ Field, field study in the western part of Japan, i.e., Niigata, Ishikawa, Toyama, Tokyo, Gunma, Nagoya, Aichi, Shiga, Kyoto, Kochi, Ehime, Shimane, Yamaguchi, and Kagoshima 
populations is shown in Table 3 . Regional differences of carrier frequency and minor allele frequency occur in the populations of Asian countries. Carrier frequency and minor allele frequency are relatively high in Korea [7, 9, 12], but low in China [7, 9, 13]. In Japan, the values are between those in Korea and China, except for in the city of Noshiro (in which the values are extremely low). Furthermore, a previous study suggested that RNF213 p.R4810K is significantly associated with systolic blood pressure [10]. Although we observed no obvious differences between GG and GA groups, additional information, such as blood pressure, should be collected from each participant in future studies. In addition, detailed symptoms and disease histories and laboratory data of subjects are needed to enable further investigation of the association of p.R4810K with other diseases in the general population.

\section{Limitations of this study}

This study has several limitations. The information about the participants' history of HT, DL, diabetes, heart disease, liver disease, kidney disease, any other disease, and drinking and smoking habits were self-reported by interview questionnaire. The sample size was relatively small and the percentage of females was large (76.87\%). Because of these limitations, it is hard to compare our data with general population data of other studies. In addition, blood pressure was not recorded in participating subjects, so our study lacks statistical power. One strength of this study is that we recorded the past and present health problems in a relatively older generation, revealing the absence of specific serious health problems. A large cohort study is needed to confirm our observations.

\begin{abstract}
Acknowledgments This study was supported by The Japan Society for the Promotion of Science KAKENHI [Grants-In-Aid for Scientific Research (A): 25253047 to AK and for Encouragement of Young Scientists (B): 15K19243 to HK] (http://www.jsps.go.jp/english/) and by a grant from the Research Committee on Spontaneous Occlusion of the circle of Willis of the Ministry of Health, Labour and Welfare of Japan (No. H26-Nanjito-Ippan-078) (http://www.mhlw.go.jp/eng lish/policy/other/research-projects/index.html). The funders had no role in study design, data collection and analysis, decision to publish, or preparation of the manuscript.
\end{abstract}

\section{Compliance with ethical standards}

Conflict of interest Prof. Koizumi has a registered patent regarding MMD: JP2010068737 'MOYAMOYA DISEASE-RELATED GENE AND UTILIZATION OF SAME'. No conflicts of interests exist for the other authors.

Ethical approval All procedures performed in studies involving human participants were in accordance with the ethical standards of the institutional and/or national research committee and with the 1964 Helsinki declaration and its later amendments or comparable ethical standards.

\section{References}

1. Suzuki J, Takaku A. Cerebrovascular, "moyamoya" disease. Disease showing abnormal net-like vessels in base of brain. Arch Neurol. 1969;20(3):288-99. doi:10.1001/archneur.1969.004800 90076012.

2. Scott RM, Smith ER. Moyamoya disease and moyamoya syndrome. N Engl J Med. 2009;360(12):1226-37. doi:10.1056/ NEJMra0804622.

3. Miao W, Zhao PL, Zhang YS, Liu HY, Chang Y, Ma J, et al. Epidemiological and clinical features of moyamoya disease in Nanjing, China. Clin Neurol Neurosurg. 2010;112(3):199-203. doi:10.1016/j.clineuro.2009.11.009.

4. Ahn IM, Park DH, Hann HJ, Kim KH, Kim HJ, Ahn HS. Incidence, prevalence, and survival of moyamoya disease in Korea: a nationwide, population-based study. Stroke. 2014;45(4):1090-5. doi:10.1161/STROKEAHA.113.004273.

5. Hoshino H, Izawa Y, Suzuki N. Epidemiological features of moyamoya disease in Japan. Neurol Med Chir (Tokyo). 2012;52(5):295-8. doi:10.2176/nmc.52.295.

6. Kamada F, Aoki Y, Narisawa A, Abe Y, Komatsuzaki S, Kikuchi $\mathrm{A}$, et al. A genome-wide association study identifies RNF213 as the first moyamoya disease gene. J Hum Genet. 2011;56(1):34-40. doi:10.1038/jhg.2010.132.

7. Liu W, Morito D, Takashima S, Mineharu Y, Kobayashi H, Hitomi T, et al. Identification of RNF213 as a susceptibility gene for moyamoya disease and its possible role in vascular development. PLoS ONE. 2011;6(7):e22542. doi:10.1371/journal. pone. 0022542 .

8. Miyatake S, Miyake N, Touho H, Nishimura-Tadaki A, Kondo Y, Okada I, et al. Homozygous c. $14576 \mathrm{G}>\mathrm{A}$ variant of RNF213 predicts early-onset and severe form of moyamoya disease. Neurology. 2012;78(11):803-10. doi:10.1212/WNL.0b013e3182 49f71f.

9. Liu W, Hitomi T, Kobayashi H, Harada KH, Koizumi A. Distribution of moyamoya disease susceptibility polymorphism p.R4810K in RNF213 in East and Southeast Asian populations. Neurol Med Chir (Tokyo). 2012;52(5):299-303. doi:10.2176/ nmc.52.299.

10. Koizumi A, Kobayashi H, Liu W, Fujii Y, Senevirathna ST, Nanayakkara S, et al. P.R4810K, a polymorphism of RNF213, the susceptibility gene for moyamoya disease, is associated with blood pressure. Environ Health Prev Med. 2013;18(2):121-9. doi:10.1007/s12199-012-0299-1.

11. Miyawaki S, Imai H, Shimizu M, Yagi S, Ono H, Mukasa A, et al. Genetic variant $R N F 213$ c. $14576 \mathrm{G}>\mathrm{A}$ in various phenotypes of intracranial major artery stenosis/occlusion. Stroke. 2013;44(10):2894-7. doi:10.1161/STROKEAHA.113.002477.

12. Jang M, Shin S, Yoon J, Ki C. Frequency of the moyamoyarelated $R N F 213$ p.Arg4810Lys variant in 1,516 Korean individuals. BMC Med Genet. 2015;16:109. doi:10.1186/s12881-0150252-4.

13. Wu Z, Jiang $\mathrm{H}$, Zhang L, Xu X, Zhang $\mathrm{X}$, Kang Z, et al. Molecular analysis of RNF213 gene for moyamoya disease in the Chinese Han population. PLoS ONE. 2012;7(10):e48179. doi:10. 1371/journal.pone.0048179. 